Journal of Clinical and Nursing Research

Research Article

\title{
Clinical Research Progress of Acupuncture and Massage Treatment in Pediatric Cerebral Palsy
}

Lin Wang ${ }^{1}$, Shijie Luo $^{2 *}$

${ }^{1}$ Shaanxi University of Chinese Medicine, Xianyang 712046, Shaanxi Province, China;

${ }^{2}$ Affiliated Hospital of Shaanxi University of Chinese Medicine, Xianyang 712000, Shaanxi Province, China

\begin{abstract}
Pediatric cerebral palsy is a common refractory disease in pediatrics, which has not only a high disability rate, but also a significant impact on the growth and daily learning of children. At present, the clinical treatment of pediatric cerebral palsy is diversified, and the curative effect is different. Among the many therapies, acupuncture and massage have the characteristics of safety, economy, and efficiency, which has unique advantages in treating the disease. However, due to the irregularity of the acupoint selection experience and manipulation, the evaluation of clinical efficacy is different. Therefore, it is necessary to summarize, analyze and organize the clinical research of acupuncture and massage treatment of pediatric cerebral palsy, in order to provide guidance and reference for clinical and follow-up research. This paper sorts out the relevant literature, and reviews the treatment of pediatric cerebral palsy from the aspects of acupuncture and massage.
\end{abstract}

Key words: Acupuncture; Massage; Pediatric cerebral palsy; Review

Publication date: September, 2020

Publication online: 30 September, 2020

*Corresponding author: Shijie Luo, luosj6666@163. com

Pediatric cerebral palsy (CP) is a non-progressive movement disorder syndrome, which is a neurodevelopmental disorder. CP is a common refractory disease in pediatrics. The incidence rate in our country is $2.0 \%-2.8 \%$, and it is increasing year by year ${ }^{[1]}$. At present, Western medicine has no specific medicines for treatment, and the cure is quite tricky. However, Chinese medicine classifies it in the categories of "five retardation", "five flaccidity", and "clonic convulsion", which are mostly caused by insufficient fetal endowment, deficiency of nephron, and insufficient vitality ${ }^{[2]}$. Pediatric cerebral palsy belongs to the theory of congenital deficiency in traditional Chinese medicine. Even pricey medicine can not make up for its deficiency, so few people treat with medicine. Acupuncture and massage, as one of the treatment methods of traditional Chinese medicine, can dredge the muscles and joints, recuperate the vitality, relieve muscle spasm, improve joint activity, enhance brain nerves, and at the same time can strengthen the spleen and stomach to improve children's digestive function and immunity, and promote the recovery of pediatric cerebral palsy. Investigations and studies have shown ${ }^{[3]}$ that the effective rate of acupuncture treatment of cerebral palsy is above $80 \%$ on average, while the effective rate of the rehabilitation training group is only $46 \%$. It shows that the therapeutic effect of acupuncture in pediatric cerebral palsy is worthy of recognition, which is beneficial to its clinical application.

\section{Clinical research of acupuncture treatment in pediatric cerebral palsy}

\subsection{Classic acupuncture}

\subsubsection{Governor vessel and Jiaji acupoint}

The physiological function of the Governor Vessel is to produce kidney qi and nurture the brain. It is said that "the disease started from the brain can be treated with the governor vessel first." Jiaji acupoints are mainly distributed on both sides of the spine and are 
the conduction pathways for sensory and motor nerve impulses. Acupuncture at the governor vessel and Jiaji acupoints can mobilize the body's Yang, dredge the meridians, adjust the qi and blood, and nourish the brain. Jiang Xianhe ${ }^{[4]}$ and others believe that acupuncture at the governor vessel and Jiaji acupoints can not only improve the physical fitness of children with cerebral palsy, but also promote the regulation of the central nervous system and improve the intelligence and exercise ability of children with cerebral palsy. Que Xiuqin $^{[5]}$ and others used acupuncture at Changqiang acupoint combined with scalp acupuncture to treat 60 cases of cerebral palsy with mental retardation, and found that the effective rate of the treatment group far exceeded that of the control group by $86 \%$. According to the analysis of the anatomical position, acupuncture at the governor vessel and Jiaji acupoints can regulate the nerves on both sides of the body, thereby promoting the intellectual and motor development of children with cerebral palsy.

\subsubsection{JIN's 3-needling}

JIN's 3-needling is a method for the treatment of pediatric cerebral palsy created by the famous Chinese medicine expert Rui Jin. There are 6 groups of acupuncture, namely Sishen-needling, Naosanneedling, Niesan-needling, Zhisan-needling, Shousanneedling and Zusan-needling. JIN's 3-needling is based on syndrome differentiation and treatment. It selects acupuncture points according to the meridian route of acupoints and modern anatomical theory, perfectly combines the holistic view of traditional Chinese medicine and the syndrome differentiation and treatment, and has a remarkable clinical effect. Haibin Yuan $^{[6]}$ found in the treatment of pediatric cerebral palsy using JIN's 3-needling treatment, that the use of JIN's 3-needling treatment can reduce arterial resistance, increase cerebral blood flow, repair damaged brain cells, and improve the intelligence of children with cerebral palsy. Yimin Zhang ${ }^{[7]}$ and others used JIN's 3-needling as the mainstay, combined acupuncture and medicine to treat 208 children with cerebral palsy. After 1 course of treatment, the total effective rate reached $90.4 \%$. JIN's 3-needling combines individualized treatment and holistic treatment into one, embodying the overall concept of Chinese medicine, and has significant clinical effects.

\subsection{Non-classical acupuncture}

\subsection{1 "Four needling for brain and abdomen"}

The Secret of Parenting Family written by Wanquan of the Ming Dynasty wrote that the physiological characteristics of the internal organs of children are--- the spleen qi is often insufficient, and the kidney qi is often weak. And the main treatment is to invigorate spleen and kidney. The four needling for brain is selected from Shenting, Baihui, and both sides of Fengchi Toufengfu to nourish the kidney, regulate cranial nerves, and nourish brain cells; the four needling for the abdomen is selected from Zhongwan, Guanyuan and Tianshu on both sides, to invigorate the spleen and stomach, replenish qi and nourish blood, strengthen and nourish marrow and essence. Guoqiang $\operatorname{Ren}^{[8]}$ collected 130 complete cases of pediatric cerebral palsy and divided them into a treatment group and a control group with 65 cases. The control group received only rehabilitation training, and the treatment group added "four needling for brain and abdomen" therapy on the basis of rehabilitation training. After one course of treatment, it was found that the scores of the GMFM-66 scale of the treatment group were significantly higher than those of the control group $(\mathrm{P}<0.05)$. The spleen and kidney are the foundation of life and growth, and the source of vital energy. The use of "four needling for brain and abdomen" therapy to invigorate the spleen and kidney can not only strengthen and nourish marrow and essence, replenish qi and nourish blood, but also enhance children's immunity and prevent diseases.

\subsubsection{Zhiqi-needling}

Zhiqi-needling is a combination of Zhisan-needling and Sishen-needling. Its acupoints are on the head, which has the effect of nourishing the brain and rejuvenating the mind. Li Huangfu ${ }^{[9]}$ selected 60 children with cerebral palsy and randomly divided them into a control group and a treatment group with 30 cases in each. The control group was treated with conventional rehabilitation plus sand table games, and the treatment group was combined with Zhiqi-needling on the basis of the control group for 3 courses. The results showed that the total effective rate of the treatment group was significantly higher than that of the control group, and the differences were statistically significant $(\mathrm{P}<0.05)$. Lin $\mathrm{Zhou}^{[10]}$ observed 46 cases of mental retardation children who received Zhiqi-needling therapy and found that Zhiqi-needling has multiple ways to improve brain function and improve children's intelligence.

\section{Massage therapy}




\subsection{Traditional massage}

Traditional massage is mostly based on the meridian and viscera. Based on the meridian theory of traditional Chinese medicine, various techniques are used to stimulate specific parts of the body surface to promote the recuperation of the vitality, dredge the meridians, and regulate the viscera. Modern medical research points out that massage can accelerate nutrient metabolism, relieve muscle spasm, repair damaged tissue, improve conduction pathways, accelerate nerve cell regeneration, and promote the recovery of brain damage in children with cerebral palsy. Yuanchun $\mathrm{Xie}^{[12]}$ randomly selected 29 children with cerebral palsy who were treated with traditional Chinese massage combined with rehabilitation training ${ }^{[11]}$. Compared with 29 children who only received rehabilitation training, the therapeutic effect of the treatment group was better than that of the control group $(\mathrm{P}<0.05)$. Yongliang Liu pointed out that early intervention of pediatric massage in children with cerebral palsy can promote brain cell recovery, reduce the rate of disability, and improve children's living quality $^{[13]}$.

\subsection{Liu's massage}

With the continuous exploration of massage therapy in the treatment of pediatric cerebral palsy, it is concluded that Liu's massage and massage for invigorating the spleen and kidney are effective in treating pediatric cerebral palsy. Liu's massage was established during the Tongzhi period of Xianfeng in Qing Dynasty. Based on children's pathological characteristics of high morbidity and rapid transmission, combined with the five elements to help and restrict each other, it forms the Five Meridian Massage to coordinate the balance of the internal organs, which is the core of Liu's massaga and is effective in the treatment of pediatric cerebral palsy ${ }^{[14]}$.

\section{Summary and expectation}

With the unique advantages, acupuncture and massage play an important role in the treatment of pediatric cerebral palsy. Compared with medicine treatment, acupuncture and massage are not only convenient, simple, safe and effective, but also can improve the general symptoms of children. However, as a traditional Chinese medical therapy, acupuncture and massage have not formulated a complete set of diagnostic criteria for traditional Chinese medicine. At the same time, there are many clinical manipulations of acupuncture and massage, which lack large samples of clinical confir- mation and effective evidence-based medical data, and the clinical efficacy is uncertain. Therefore, improving the existing technology and its combination to produce new technical strategies to provide new solutions for the treatment of $\mathrm{CP}$ is an area that medical researchers should focus on. At present, the widely concerned fivetone therapy combined acupuncture and massage with AI technology can develop a new type of treatment instrument for cerebral palsy and provide a new perspective for CP treatment. As medical students, we should continue to explore a standardized treatment plan that is more satisfying and in line with pediatric cerebral palsy based on new techniques derived from acupuncture and massage.

\section{References}

[1] Jing P, Wang XW, Sun DM, etc. Research progress of core stability training $[\mathrm{J}]$. Chinese Rehabilitation Theory and Practice, 2014, 20(7): 629-633.

[2] Wang YQ. Application of Liu's Rongdu Jiu-point Mofang in Pediatric Cerebral Palsy[J]. Chinese Folk Therapy, 2019, 27(19): 36-38.

[3] Qi YC, Liu ZH. Current status and prospects of treatment of cerebral palsy in children[J]. Chinese Journal of Practical Nervous Diseases, 2006(1): 102-105.

[4] Jiang XH, Zhang JK, Ma BX, et al. Application of acupuncture and massage of governor vessel and Jiaji acupoints in children with cerebral palsy[J]. Pediatrics of Chinese Medicine, 2019, 15(3): 82-86.

[5] Que XQ, Li XL, Wu CF, et al. Acupuncture at Changqiang acupoint combined with scalp acupuncture to treat 30 cases of children with cerebral palsy with mental retardation[J]. Fujian Traditional Chinese Medicine, 2018, 49(6): 73-74+77.

[6] Li YH. A clinical study on the treatment of cognitive dysfunction in children with cerebral palsy by acupuncture method for regulating the mind and supervising supervising skills combined with cognitive function training[D]. Hubei University of Traditional Chinese Medicine, 2016.

[7] Zhang YM, Rui J, Zhang XL, et al. Treatment of 208 cases of children with cerebral palsy with "Jin's three-needling" as main acupuncture combined with medicine[J]. Hunan Journal of Traditional Chinese Medicine, 2000(7): 23-24.

[8] Wang DJ, Chen ZW, Du QX, et al. Under the guidance of Ziwu Liuzhu theory. Jin's three-needling combined with oral Chinese medicine for the treatment of 46 cases of children with cerebral palsy. Clinical observation on Jin's three-needling combined with massage in the treatment of children with cerebral palsy[J]. 19(2): 145-146.

[9] Ren GQ. Zhou Zhijie's academic thoughts and the clinical study of "brain and abdomen four needles" in the treatment of children with spastic cerebral palsy [D]. China Academy of Chinese Medical Sciences, 2016.

[10] Tu YD, Gong CX, He ZQ. Gong Changxiang's clinical experi- 
ence in the treatment of cerebral palsy with Gong's brain acupuncture $[\mathrm{J}]$. Liaoning Journal of Traditional Chinese Medicine, 2019, 46(6): 1156-1158.

[11] Zhou L. Analysis of the effect of Zhiqi-acupuncture combined with rehabilitation training on improving the intelligence and cognitive ability of children with mental retardation[J]. Journal of Acupuncture and Moxibustion, 2017, 33(12): 12-15.

[12] Wei XX, Lei LM, Zhang HY, et al. Overview of the application of massage therapy in the clinical rehabilitation of children with cerebral palsy[J]. Journal of Shandong University of Traditional Chinese Medicine, 2019, 43(3): 313-316.

[13] Liu YL. Observation on the curative effect of early intervention of pediatric massage in the treatment of cerebral palsy[J]. Massage and Rehabilitation Medicine, 2017, 8(20): 38-40.
[14] Li X, Shao XN. Liu's principles of pediatric massage with points and views of monarchs and ministers[J]. Shizhen Traditional Chinese Medicine and Materia Medica, 2017, 28(9): 2195-2196.

[15] Su HB. The therapeutic effect of Liu's pediatric massage on children with cerebral palsy with mild intellectual disability and its influence on the patient's intelligence and exercise level [J]. Shaanxi Journal of Traditional Chinese Medicine, 2018, 39(10): 1470-1472+1475

[16] Yi XC, Li XR, Hao PP, et al. Observation on the clinical efficacy of Liu's pediatric massage in the treatment of 38 cases of cerebral palsy[J]. Herald of Chinese Medicine, 2016, 22(9): 4751. 